1. E. B. Escotr, "Amicable numbers," Scripta Math., v. 12, 1946, pp. 61-72. MR 8, 135. p. 77.

2. P. Poulet, "Forty-three new couples of amicable numbers," Scripta Math., v. 14, 1948,

3. M. Garcia, "New amicable pairs," Scripta Math., v. 23, 1957, pp. 167-171. MR 20 *5158.

4. L. E. Dickson, "Theorems and tables on the sum of the divisors of a number," Quart. J. Pure Appl. Math., v. 44, 1913, pp. 264-296.

5. O. ORE, Number Theory and Its History, McGraw-IIill, New York, 1948. MR 10, 100.

6. L. E. Dickson, History of the Theory of Numbers, Carnegie Institution Publ. no. 256, 1919; reprint, G. E. Stechert, 1934.

7. D. H. Lenmer, Guide to Tables in the Theory of Numbers, Bull. Nat. Research Council, no. 105, National Research Council, Washington, D. C., 1941. MR 2, 247.

8. P. Poulet, La Chasse aux Nombres, Vol. 1, Stevens, Brussels, 1929.

\title{
Primes of the Form $n^{4}+1$
}

\author{
By M. Lal
}

In this note we report 172 new primes of the form $n^{4}+1$ and tabulate all such primes for $1 \leqq n \leqq 4004$.

Factorization of the numbers of the form $n^{4}+1$ has been extensively studied by Cunningham [1] and Gloden [2], [3]. They used a sieve method based on the four solutions of the congruence equation

$$
x^{4}+1 \equiv 0 \quad(\bmod p)
$$

for all primes of the form $8 k+1$. With primes less than $4 \times 10^{6}$, numbers $n^{4}+1$ for $n \leqq 2000$ have been completely factorized.

For $p>4 \times 10^{6}$, it becomes rather difficult and time consuming to solve (1). Consequently, it renders such a sieve less practical. An analysis of the growing inefficiency of such a sieve with increasing $p$ is given in $[5, \mathrm{p}$. 188]. However, the range for $n$ can be extended by using Alway's method [4] of factorization modified for odd divisors of the form $8 k+1$ and testing each number $n^{4}+1$ individually.

With the modified Alway's method, we found all primes for $1 \leqq n \leqq 1000$. This was done to check the program and to provide independent data which is not readily accessible for this interval. The search was then extended to $2000 \leqq n \leqq$ 4004; 172 primes and one prime factor for other composite numbers were indentified. The time required to establish the primality of $n=4002$ is 2.0 hours on the IBM 1620 computer Model II. As the project required several hundred hours of machine time, the search was made on three IBM 1620 computers-one at Memorial and two at Kingston. All primes of the form $n^{4}+1$, complemented by those given in [3] for $1000<n<2040$, are presented in Table 1 .

Discussion of Results. Shanks [5] has made a conjecture regarding the number of primes $Q(N)$ of the form $n^{4}+1$ for $1 \leqq n \leqq N$ and has given the following expression:

$$
Q(N) \sim .66974 \int_{2}^{N} \frac{d n}{\log n} .
$$

The observed count and those computed by using (2), rounded to the first decimal, are given below in Table 2. 
TABLE $1^{*}$

Primes of the form $n^{4}+1$

\begin{tabular}{|c|c|c|c|c|c|c|c|c|c|}
\hline 1 & 48 & 132 & 204 & 276 & 364 & 492 & 566 & 702 & 772 \\
\hline 2 & 54 & 140 & 210 & 278 & 374 & 494 & 568 & 710 & 778 \\
\hline 4 & 56 & 142 & 220 & 288 & 414 & 498 & 582 & 730 & 786 \\
\hline 6 & 74 & 154 & 228 & 296 & 430 & 504 & 584 & 732 & 788 \\
\hline 16 & 80 & 160 & 238 & 312 & 436 & 516 & 600 & 738 & 798 \\
\hline 20 & 82 & 164 & 242 & 320 & 442 & 526 & 616 & 742 & 800 \\
\hline 24 & 88 & 174 & 248 & 328 & 466 & 540 & 624 & 748 & 810 \\
\hline 28 & 90 & 180 & 254 & 334 & 472 & 550 & 628 & 758 & 856 \\
\hline 34 & 106 & 194 & 266 & 340 & 476 & 554 & 656 & 760 & 874 \\
\hline 46 & 118 & 198 & 272 & 352 & 488 & 556 & 690 & 768 & 894 \\
\hline 912 & 996 & 1144 & 1246 & 1404 & 1536 & 1610 & 1680 & 1788 & 1910 \\
\hline 914 & 1038 & 1150 & 1252 & 1406 & 1540 & 1612 & 1688 & 1806 & 1916 \\
\hline 928 & 1042 & 1152 & 1270 & 1428 & 1542 & 1618 & 1700 & 1820 & 1926 \\
\hline 930 & 1072 & 1170 & 1280 & 1434 & 1552 & 1622 & 1706 & 1824 & 1932 \\
\hline 936 & 1076 & 1180 & 1302 & 1442 & 1554 & 1638 & 1710 & 1836 & 1934 \\
\hline 952 & 1088 & 1200 & 1322 & 1446 & 1558 & 1644 & 1718 & 1850 & 1942 \\
\hline 962 & 1126 & 1202 & 1330 & 1458 & 1568 & 1646 & 1722 & 1854 & 1944 \\
\hline 966 & 1132 & 1218 & 1344 & 1472 & 1586 & 1650 & 1738 & 1864 & 1948 \\
\hline 986 & 1136 & 1236 & 1382 & 1486 & 1594 & 1652 & 1754 & 1870 & 1952 \\
\hline 992 & 1142 & 1238 & 1388 & 1496 & 1598 & 1666 & 1772 & 1892 & 1956 \\
\hline 1962 & 2074 & 2204 & 2260 & 2374 & 2478 & 2560 & 2674 & 2770 & 2964 \\
\hline 1972 & 2102 & 2206 & 2266 & 2376 & 2482 & 2578 & 2676 & 2798 & 2998 \\
\hline 1978 & 2104 & 2222 & 2292 & 2384 & 2486 & 2590 & 2690 & 2804 & 3006 \\
\hline 1986 & 2108 & 2224 & 2296 & 2400 & 2488 & 2598 & 2698 & 2834 & 3012 \\
\hline 1994 & 2126 & 2226 & 2312 & 2408 & 2510 & 2604 & 2700 & 2866 & 3022 \\
\hline 2040 & 2142 & 2238 & 2322 & 2414 & 2512 & 2612 & 2724 & 2872 & 3030 \\
\hline 2044 & 2152 & 2240 & 2336 & 2428 & 2522 & 2622 & 2732 & 2876 & 3046 \\
\hline 2046 & 2158 & 2250 & 2350 & 2432 & 2536 & 2640 & 2734 & 2902 & 3070 \\
\hline 2058 & 2162 & 2254 & 2360 & 2436 & 2546 & 2642 & 2736 & 2936 & 3084 \\
\hline 2068 & 2192 & 2256 & 2368 & 2438 & 2554 & 2646 & 2740 & 2958 & 3090 \\
\hline 3094 & 3246 & 3410 & 3502 & 3626 & 3720 & 3862 & 3972 & & \\
\hline 3100 & 3254 & 3416 & 3516 & 3632 & 3752 & 3870 & 3982 & & \\
\hline 3104 & 3268 & 3422 & 3522 & 3642 & 3756 & 3872 & 3988 & & \\
\hline 3108 & 3286 & 3450 & 3530 & 3644 & 3764 & 3882 & 3992 & & \\
\hline 3124 & 3288 & 3456 & 3550 & 3666 & 3780 & 3896 & 3998 & & \\
\hline 3128 & 3322 & 3464 & 3574 & 3688 & 3796 & 3910 & 4000 & & \\
\hline 3132 & 3326 & 3468 & 3576 & 3692 & 3802 & 3926 & 4002 & & \\
\hline 3162 & 3378 & 3472 & 3586 & 3700 & 3842 & 3954 & & & \\
\hline 3200 & 3386 & 3480 & 3618 & 3702 & 3854 & 3958 & & & \\
\hline 3244 & 3390 & 3492 & 3620 & 3718 & 3856 & 3960 & & & \\
\hline
\end{tabular}

* I am indebted to the late Professor Albert Gloden for his kind permission to include his results for $1000<n<2040$.

The agreement between the actual and computed counts is remarkably good. From Table 1, one observes that "twin" primes (those where $n^{4}+1$ and $(n+2)^{4}+$ 1 are both primes) occur quite frequently. The number of such twins $P(N, N+2)$ for $1 \leqq n \leqq N$ is given in Table 3 .

On the basis of heuristic arguments [6], one would expect the number 
TABLE 2

\begin{tabular}{r|r|r}
\hline$N$ & \multicolumn{2}{|c}{ Counts } \\
\cline { 2 - 3 } & Observed & Computed \\
\hline 500 & 63 & 67.5 \\
1000 & 111 & 118.3 \\
1500 & 150 & 165.3 \\
2000 & 205 & 210.1 \\
2500 & 254 & 253.5 \\
3000 & 292 & 295.8 \\
3500 & 330 & 337.3 \\
4000 & 376 & 377.9 \\
\hline
\end{tabular}

TABLE 3

Twin primes

\begin{tabular}{c|c|c|c}
\hline$N$ & $P(N, N+2)$ & $R=N /(\log N)^{2}$ & $P(N, N+2) / R$ \\
\hline 1000 & 18 & 20.96 & .86 \\
2000 & 30 & 34.62 & .87 \\
3000 & 46 & 46.80 & .98 \\
4000 & 58 & 58.15 & 1.00 \\
\hline
\end{tabular}

$P(N, N+2)$ to be proportional to $N /(\log N)^{2}$ and it is gratifying to note that this is indicated in column 4 above. These twins are analogous to the Gaussian twin primes examined in [7].

Acknowledgments. I wish to thank the Directors of the Computing Centre of Queen's University and the Royal Military College, Kingston, for the free use of their computers. Thanks are also due to the Canadian Mathematical Congress for the award of a Summer Research Fellowship.

Department of Mathematics

Memorial University of Newfoundland

St. John's, Newfoundland, Canada

1. A. J. C. Cunningham, Binomial Factorizations, Vols. I, IV, Francis Hodgson, London, 1923.

2. A. Gloden, "Additions to Cunningham's factor table of $n^{4}+1$," Math. Comp., v. 16, 1962 , pp. 239-241. MR 26 *2384.

3. A. Gloden, "New primes of the form $n^{4}+1$," Math. Comp., v. 19, 1965, pp. 144-145. MR 30 * 1105.

4. G. G. Alway, "A method of factorization using a high-speed computer," MTAC, v. 6, 1952 , pp. $59-60$.

5. D. Shanks, "On numbers of the form $n^{4}+1$," Math. Comp., v. 15, 1961, pp. 186-189; Corrigendum, ibid., v. 16,1962 , p. 513. MR 22 * 10941 .

6. P. T. BATEMAN \& R. A. HoRn, "A heuristic asymptotic formula concerning the distribution of prime numbers," Math. Comp., v. 16, 1962, pp. 363-367. MR 26 *6139.

7. D. Shanks, "A note on Gaussian twin primes," Math. Comp., v. 14, 1960, pp. 201-203. MR 22 *2586. 